\title{
CONGRUENCIA EN CONOCIMIENTOS Y EDUCACIÓN AFECTIVO-SEXUAL EN PERSONAS CON DI
}

\author{
Lorena Caballero-Gascón \\ Dpto. Psicología Evolutiva y de la Educación. \\ Salusex-Unisexsida. Universitat de València, Estudi General. \\ lorena.caballero@uv.es \\ Cristina Giménez-García \\ Dpto. Psicología Básica, Clínica y Psicobiología. \\ Salusex-Unisexsida. Universitat de Jaume I. \\ Jesús Castro-Calvo \\ Dpto. Psicología Básica, Clínica y Psicobiología. \\ Salusex-Unisexsida. Universitat de Jaume I. \\ Addictive and Compulsive Behaviours Lab, Institute for Health and Behaviour, \\ University of Luxembourg, Esch-sur-Alzette, Luxembourg. \\ Marta García Barba \\ Dpto. Psicología Básica, Clínica y Psicobiología. \\ Salusex-Unisexsida. Universitat de Jaume I. \\ $M^{\mathrm{a}}$ Dolores Gil-Llario \\ Dpto. Psicología Evolutiva y de la Educación. \\ Salusex-Unisexsida. Universitat de València, Estudi General.
}

Fecha de Recepción: 27 Febrero 2018

Fecha de Admisión: 10 Abril 2018

\section{RESUMEN}

En la educación de las personas con diversidad funcional intelectual (DFI) es importante que los principales agentes implicados (usuarios/as, padres y profesionales que trabajan con ellos) posean criterios comunes acerca de los objetivos educativos en general, y especialmente, en el área de la sexualidad. Sin embargo, las tres perspectivas no siempre son coincidentes con las consecuencias que se derivan de ello. Teniendo en cuenta la importancia de la identificación de necesidades y metas comunes, planteamos este trabajo con el objetivo de conocer el grado de concordancia entre: 1) los conocimientos que los usuarios tienen sobre sexualidad y la que los padres y profesionales les atribuyen y 2) la opinión de padres, profesionales y usuarios/as sobre la necesidad de proveer/recibir educación afectivo-sexual. Para ello, 92 adultos con DI (45.6\% mujeres, 54.4\% hombres) con edades entre los 19 y 67 años $(M=37.5)$ y sus padres y profesionales, completaron un instrumento donde se les preguntaba acerca del conocimiento sobre sexualidad y sobre el interés de recibir formación en educación afectivo-sexual. Los resultados señalan una concordancia respecto a los conocimientos sobre sexualidad del $36.7 \%$ entre padres y usuarios/as y del $58.4 \%$ entre profesionales y usuarios. En cuanto a la concordancia respecto a la necesidad de recibir formación en educación afectivo-sexual, ésta fue $75.5 \%$ entre padres y usuarios/as y del $88.5 \%$ entre profesionales y usuarios. La concordancia entre los tres respecto a esta cuestión resultó alta (74.7\%). A partir de estos resultados podemos concluir que los profesionales son mejores conocedores de las necesidad, capacidades y conocimientos de los/as usuarios/as de lo que lo son sus propios padres pero que todos tienen en común el identificar la necesidad de introducir de forma normalizada la educación afectivo-sexual en este colectivo. 


\section{CONGRUENCIA EN CONOCIMIENTOS Y EDUCACIÓN AFECTIVO-SEXUAL EN PERSONAS CON DI}

Palabras clave: congruencia; conocimientos en sexualidad; educación afectivo-sexual; adultos con DI; padres; profesionales

\section{ABSTRACT}

Knowledge and affective-sexual education congruence in people with id.

In education of people with intellectual disability (ID) it is important that the main agents involved (users, parents and professionals working with them) have common criteria about educational objectives in general, and especially, in sexuality. However, the three perspectives do not always coincide with the consequences of it. Taking into account the importance of identifying common needs and goals, we propose this work with the objective of knowing the degree of agreement between: 1) the knowledge that users have about sexuality and the attribution their parents and professionals made about them and 2) the opinion of parents, professionals and users on the need to provide/receive affective-sexual education. For this, 92 adults with ID (45.6\% women, $54.4 \%$ men) with ages between 19 and 67 year old $(M=37.5)$ and their parents and professionals, completed an instrument where they were asked about knowledge about sexuality and the interest of receiving training in affective-sexual education. Results indicate agreement respect to sexuality knowledge of $36.7 \%$ between parents and users and $58.4 \%$ between professionals and users. Regarding the agreement of the need to receive training in affective-sexual education, this was $75.5 \%$ between parents and users and $88.5 \%$ between professionals and users. The agreement between the three regarding this issue was high $(74.7 \%)$. From these results we can conclude that professionals are better aware of the needs, skills and knowledge of the users than their own parents are but, all have in common the identification of the need to introduce in a standardized way affective-sexual education in this group.

Keywords: congruence; sexuality knowledge; affective-sexual education; ID adults; parents; professionals

\section{ANTECEDENTES}

El objetivo de educar, es contribuir a la adquisición de todos aquellos conocimientos, habilidades y actitudes necesarios para el óptimo desarrollo del ser humano. Aunque la educación es necesaria en todos los ámbitos de la vida, la referida a los aspectos sexuales frecuentemente es evitada. Este fenómeno se agudiza en el colectivo con diversidad funcional intelectual (de ahora en adelante DFI). Por ello, el limitado conocimiento sexual de las personas con DFI constituye una de las barreras principales con que se encuentra este grupo (Schaafsma, Kok, Stoffelen, \& Curfs, 2014; Schwartz \& Robertson, 2018; Thompson, Stancliffe, Broom, \& Wilson, 2014, 2016). Este hecho se debe a diversas causas: falta de conocimientos por parte de padres y formadores para abordar las explicaciones ya desde la adolescencia (Evans, McGuire, Healy, \& Carley, 2009; Lafferty, McConkey, \& Simpson, 2012); actitudes paternalistas, sobreprotectoras, negativas, prohibitivas y reguladoras -entre otras- del entorno cercano de las personas con DI; limitadas posibilidades de establecer relaciones personales y sexuales así como la falta de privacidad (Evans et al., 2009); educación sexual limitada a temas relacionados con la prevención de embarazos, ITS y abuso sexual (Schwartz \& Robertson, 2018) y otras descritas en la literatura.

Las personas con DFI, tienen curiosidades, sentimientos y necesidades sexuales como cualquier otra persona por lo que, estas trabas no hacen sino impedir su óptimo desarrollo psicosexual (Lotta Löfgren-Mårtenson, 2004). Cuanto menor sea su conocimiento en educación sexual, mayor será la probabilidad de desarrollar actitudes negativas hacia la sexualidad, embarazos no deseados, dificultad para distinguir entre espacios públicos y privados y menor experiencia en el área de las relaciones sexuales (McCabe \& Cummins, 1996). Tal es así que, en un estudio llevado a cabo por Löfgren- 
Mårtenson (2012) en el que se entrevistaron a 16 personas con DFI entre 16 y 21 años, demostraron que apenas recordaban haber recibido educación sexual, o que, en caso afirmativo, habían encontrado dificultades para entender lo que se les explicaba.

Las actitudes mostradas por familiares, cuidadores y profesionales son en muchas ocasiones conservadoras (Cuskelly \& Bryde, 2004; Povilaitiene \& Radzeviciene, 2013), y, por tanto, negativas ya sea por vergüenza, por no saber cómo explicar temas sexuales o por miedo a "despertar" conductas inapropiadas (Rohleder, 2010). Estas reticencias provocan que las personas con DFI busquen información por otros medios (amigos, televisión, revistas, internet, etc.) (Schwartz \& Robertson, 2018), adquiriendo modelos en la mayoría de casos, poco adecuados. De ello deriva la importancia de contribuir a la necesidad de fomentar actitudes positivas hacia la sexualidad en el entorno de las personas con DFI, que sean favorables a la aplicación de programas de educación afectivo-sexual para adquirir conocimientos apropiados (Schaafsma et al., 2014).

Los padres son los primeros educadores sexuales y por ello deberían proveer de conocimientos a sus hijos y resolver las dudas que les cuestionan respecto a este tema. Isler, Beytut, Tas y Conk (2009) documentaron que un $32.5 \%$ de los padres nunca había hablado de sexualidad con sus hijos y un $42.5 \%$ no les había ofrecido ningún tipo de educación afectivo-sexual. Por otro lado, cuando se les preguntaba a las propias personas con DFI, un $37.5 \%$ había hablado de sexualidad con sus madres y un $67.5 \%$ dijeron haber compartido sus experiencias sexuales con ellos. Thompson, Stancliffe, Broom y Wilson (2014) observaron que los profesionales que trabajan con las personas con DFI cada vez tienen actitudes más positivas hacia su salud sexual; sin embargo, habitualmente se encuentran con dificultades para poder proveer de esta educación a sus usuarios/as por miedo a que los padres les impidan finalizar esas formaciones.

El entorno cercano a las personas con DFI suele valorar de manera muy pobre sus capacidades, atribuyéndoles menos conocimientos de los que realmente disponen. De esas actitudes dependerá que se haga mayor o menor hincapié en la necesidad de proporcionar educación afectivo-sexual, reduciendo así la probabilidad de que se cumplan todas aquellas preocupaciones que habitualmente padecen padres y profesionales (p.e. que el usuario sea víctima de abuso sexual, de un embarazo no deseado, etc.) (Byrne, 2017). De ello deriva el interés de este estudio con un doble objetivo: 1) constatar la congruencia de los conocimientos que los/as usuarios/as tienen sobre sexualidad y la que los padres y profesionales les atribuyen; y 2) observar si hay congruencia entre la opinión de padres, profesionales y usuarios/as sobre la necesidad de proveer/recibir educación afectivo-sexual.

\section{MÉTODO}

\section{Participantes}

En este trabajo se evaluaron un total de 276 participantes clasificados en tres grupos: adultos/as con DFI (92 participantes), sus padres, madres o tutores/as legales (92 participantes) y los/as profesionales que trabajan con ellos/as en los centros ocupacionales a los que asisten (92 participantes). Los 92 adultos con DFI evaluados (45.6\% mujeres; $54.4 \%$ hombres) tenían edades comprendidas entre los 19 y los 67 años ( $M=37.5 ; D . T=9.72)$. En cuanto a su nivel intelectual, el $76.7 \%$ presentaba discapacidad intelectual moderada y el $20.5 \%$ leve. Atendiendo al lugar de residencia, la mayoría de ellos vivía en el domicilio familiar (92.4\%), seguido de residencias (3.3\%) y por último en viviendas tuteladas 0 independizados (2.2\% respectivamente).

\section{Instrumentos}

Escala de Comportamiento Sexual (ECS, Gil, Ballester y Morell, 2017) en sus versiones para padres (ECS-PA), profesionales (ECS-PR) y autoinforme del usuario (ECS-A). En ellos se combinan 


\section{CONGRUENCIA EN CONOCIMIENTOS Y EDUCACIÓN AFECTIVO-SEXUAL EN PERSONAS CON DI}

preguntas con diferentes formatos de respuesta (ítems dicotómicos, respuesta múltiple, tipo Likert) ajustadas a cada colectivo. El ECS en sus tres versiones incluye preguntas comunes que permiten contrastar la opinión entre las tres fuentes de información. De ahí se extrajeron los ítems utilizados en este estudio.

De la versión ECS-A (usuarios), se usaron los ítems: “¿Crees que la información que tienes sobre sexo es correcta?” " “Te gustaría que te dieran clase sobre qué es la sexualidad, cómo actuar en situaciones íntimas, etc.?”. De la versión ECS-PR (profesionales): “¿Piensa que el/la usuario/a tiene algún conocimiento sobre sexualidad?” y “ ¿Se beneficiaría el/la usuario/a de una adecuada educación afectivo-sexual?". Finalmente, de la versión ECS-PA (padres) se usaron: "¿Piensa que su hijo/a tiene conocimientos sobre la sexualidad?" y "¿Se beneficiaría su hijo de una adecuada educación afectivo-sexual?". En todos los casos, el formato de respuesta a estas preguntas fue dicotómico (sí/no).

\section{Procedimiento}

En primer lugar, se estableció contacto con la Asociación Coordinadora de Centros Ocupacionales de la Comunidad Valenciana (COPAVA), que dio su consentimiento para la recogida de datos en varios de sus centros. Seguidamente se informó a las personas con DFI que cumplían los criterios de inclusión en el estudio del objetivo de la investigación y se pidió su consentimiento para participar. Los criterios de inclusión fueron: 1) tener más de 18 años; 2) cumplir los criterios del DSM-5 para Discapacidad Intelectual (leve o moderada) y; 3 ) tener suficientes habilidades de comunicación y lectura para poder responder de forma fiable a las cuestiones planteadas.

La evaluación fue llevada a cabo por dos miembros de SALUSEX con experiencia en evaluación e intervención en personas con DFI. Cada participante fue evaluado individualmente. Los que mostraban buen desempeño en lectura, recibieron una breve explicación sobre cómo completar la escala y fueron guiados durante el proceso de evaluación atendiendo a las dudas cuando se producían. Los que no poseían competencia lectora cumplimentaron la evaluación a través de una entrevista en la que los investigadores iban leyendo y explicando, cuando así se precisaba, las preguntas de los cuestionarios. El tiempo promedio de evaluación fue de unos 15-20 minutos. Antes de comenzar se advirtió a los participantes que sus respuestas eran totalmente anónimas y confidenciales.

Los padres y profesionales cumplimentaron sus respectivos cuestionarios pensando en su hijo/a en el caso de padres o en el/la usuario/a en particular en el caso de los/as profesionales.

\section{Análisis de datos}

Los datos se analizaron a través del software SPSS versión 24.0. En primer lugar, se realizaron análisis descriptivos (medias y desviaciones típicas) para explorar las características clínicas y socio-demográficas de los/as adultos/as con DFI. Posteriormente, se realizaron tablas de contingencia para comprobar la congruencia entre usuarios, padres y profesionales.

\section{RESULTADOS}

El primer nivel de análisis se centró en la opinión acerca de los conocimientos sobre sexualidad. Como podemos observar en la figura 1 , un $63.6 \%$ de los usuarios con DFI consideraba que sus conocimientos sobre sexo eran adecuados, un $58.8 \%$ de los padres pensaba que sus hijos/as tenían conocimientos adecuados y suficientes sobre sexualidad ascendiendo esta cifra a un $83.6 \%$ en el grupo de los profesionales. 
Figura 1. Conocimientos sobre sexualidad

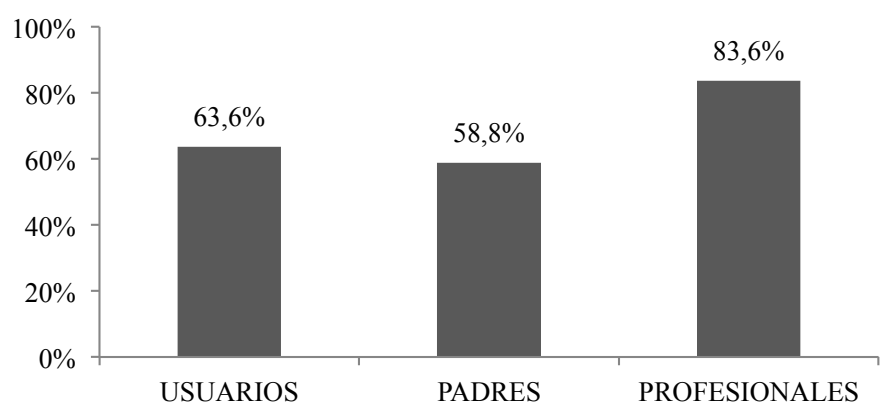

Por lo que se refiere a la opinión acerca de los beneficios de recibir (usuarios) o proporcionar (padres y profesionales) educación afectivo-sexual (ver figura 2), un $90.5 \%$ de los usuarios consideraba positivamente recibir formación en sexualidad frente a tan sólo un 9.5\% que opinaba lo contrario. En cuanto al aprovechamiento por parte de los/as hijos/as con DFI de educación afectivosexual, un $76.2 \%$ de los padres pensaba que sus hijos/as se beneficiarían de ello. Finalmente, casi todos los profesionales $(97.7 \%)$, consideraban beneficioso que sus usuarios recibieran educaciónafectivo sexual.

Figura 2. Recibir/proporcionar Educacion Afectivo-Sexual.

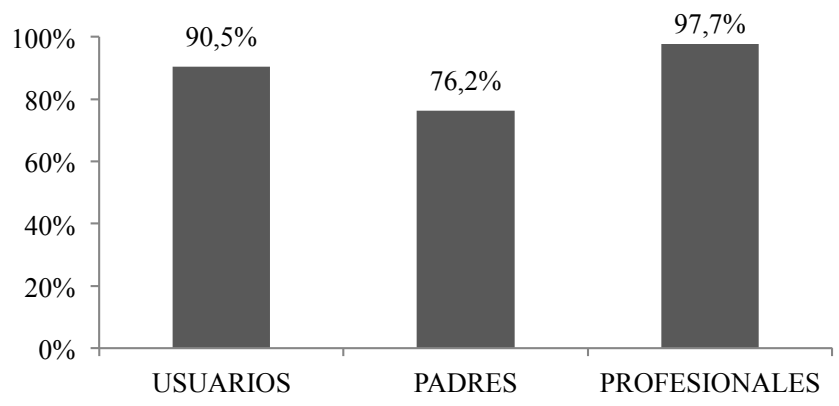

Tras analizar de manera individual la opinión de cada grupo de participantes respecto a las dos cuestiones objeto de estudio, se exploró el grado de congruencia entre la opinión de los 3 agentes implicados. Con respecto a la pregunta de si los usuarios disponían de conocimientos suficientes y adecuados sobre sexo, la opinión de los padres y la de los usuarios tan sólo coincidió en el $36.7 \%$ de los casos (ver figura 3), eso significa que en el 63.3\% restante, los padres consideraban que sus hijos no tenían los conocimientos suficientes sobre sexo cuando ellos mismos consideraban que sí y viceversa. Este grado de congruencia ascendía al $54.2 \%$ cuando se comparaba la opinión de los padres y profesionales y al $58.4 \%$ (el porcentaje de congruencia más alto) cuando se comparaba a usuarios y profesionales. Finalmente, sólo en el $35.9 \%$ de casos los 3 grupos coincidían en valorar positivamente los conocimientos sobre sexualidad. 
Figura 3. Congruencia usuarios-padres-profesionales en conocimientos sobre sexualidad

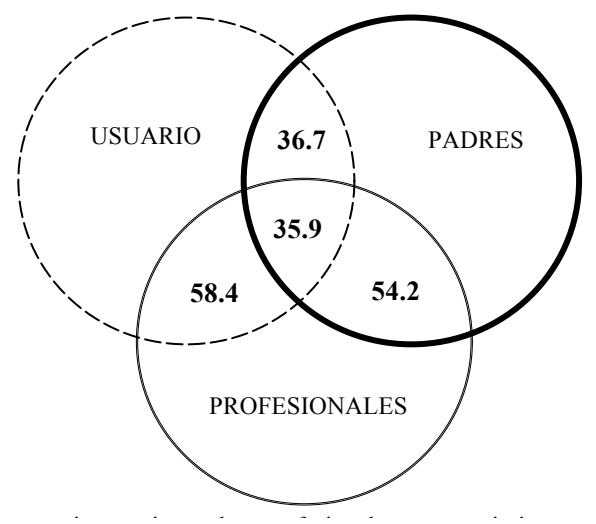

Finalmente, se exploró el grado de congruencia en cuanto al beneficio de proporcionar/recibir educación afectivo-sexual (ver figura 4). En el $75.5 \%$ de los casos, usuarios y padres compartían la necesidad que se realizara formación en sexualidad o lo que es lo mismo, sólo en el $24.5 \%$ de los casos, los padres no valoraban positivamente esta necesidad cuando en realidad sus hijos sí lo creían necesario y viceversa. Nuevamente, la congruencia aumentaba ligeramente cuando se exploraba la opinión de padres y profesionales (76.3\%) y alcanzaba su valor más alto cuando se contrastaba la opinión de usuarios y profesionales (88.5\%). Así, profesionales y usuarios únicamente discrepaban en el $11.5 \%$ de los casos. Finalmente, la congruencia entre la opinión de los tres agentes respecto a esta cuestión fue del $74.7 \%$.

Figura 4. Congruencia usuarios-padres-profesionales en educacion afectivo-sexual

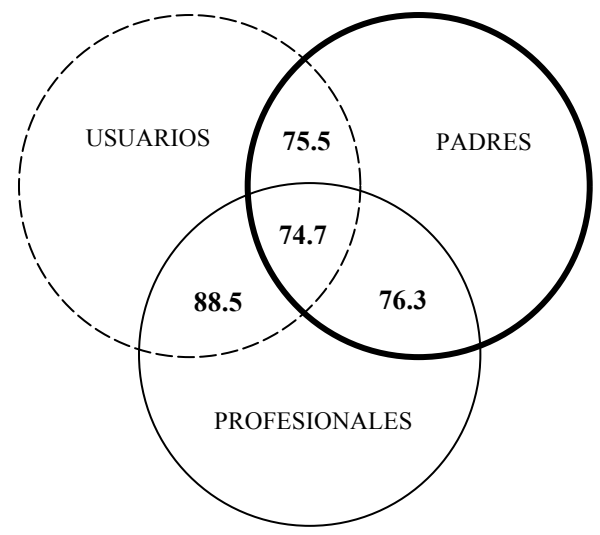

\section{DISCUSIÓN Y CONCLUSIONES}

A la vista de los resultados podemos afirmar que, en general, hay un bajo grado de congruencia entre los 3 grupos en cuanto al nivel de conocimientos sobre sexo atribuidos a las personas con DFI. Por otro lado, Ios resultados muestran una tendencia contraria en cuanto al beneficio de reci- 
bir/proporcionar educación afectivo-sexual, Ilegando a un porcentaje considerablemente alto en esta cuestión.

Analizándolos por separado, en cuanto a la pregunta relacionada con los conocimientos sobre sexo, el grupo de profesionales es el que mejor valora a los/as usuarios/as (83.6\%). Este resultado es seguido por el de los propios usuarios y finalmente por el grupo de padres. La buena actitud de los profesionales, puede deberse a que este grupo conoce muy bien a los usuarios que asisten a sus centros ocupacionales así como las dinámicas que se siguen en ellos. Además, los profesionales tienden cada vez más a formar sus usuarios/as en todos los aspectos y, por lo tanto, les atribuyen un buen nivel de conocimientos sobre sexualidad. Estos resultados contrastan con los obtenidos por los padres que siguen manteniendo actitudes reticentes a tratar el tema de la sexualidad, tal como otros autores han señalado (Aunos \& Feldman, 2002) y, por ello, es el grupo que peor valora los conocimientos de sus hijos/as. Finalmente, los/as usuarios/as valoran positivamente sus conocimientos sobre sexualidad. Esto puede deberse a una tendencia a la desmitificación del tema de la sexualidad y a poder abrirse y hablar de estos temas en determinadas circunstancias.

Continuando con la interpretación de los resultados por separado, en la segunda cuestión (beneficios de la educación afectivo-sexual) se muestran actitudes muy positivas. Nuevamente, el grupo de profesionales es el que considera más beneficiosa la incorporación de esta formación, seguidos de los/as usuarios/as y finalmente por el grupo de padres. Estas buenas actitudes (sobre todo en el grupo de los padres) pueden deberse a la consideración de que si la educación en sexualidad es buena para todo el mundo, también debe serlo para sus hijos/as. Otra posible interpretación es que, al intentar tratar esta dimensión con mayor "normalidad", se abogue por la educación afectivosexual como medio para solventar dudas, necesidades, problemas, etc., que acontecen en el día a día de cualquier persona y también en el de las personas con DFI (Azzopardi-Lane \& Callus, 2015; Frawley \& Wilson, 2016).

Un segundo nivel de análisis conjunto dirigido a identificar el grado de congruencia entre los tres grupos, permite concluir que hay una baja coincidencia de opiniones en cuanto a la atribución de conocimientos sobre sexo en las personas con DFI. Sin embargo, los datos muestran una alta congruencia en cuanto al beneficio de programas de educación afectivo-sexual para los/as usuarios/as. Estos datos van en la línea de los observados en otras investigaciones (Schaafsma et al., 2014; Schwartz \& Robertson, 2018). En cuanto a la primera cuestión (conocimientos sobre sexo), la mayor coincidencia la encontramos entre el grupo de usuarios/as y profesionales. Esto quiere decir que, cuando los/as usuarios/as consideran que los conocimientos sobre sexo que tienen son adecuados, los profesionales están de acuerdo con ello y viceversa. El grado de congruencia más baja se encuentra entre el grupo de padres y el de usuarios/as, esto es, cuando ellos/as consideran que tienen suficientes conocimientos sobre sexo, pocos son los padres que consideran que sus hijos/as los tienen (36.7\%). El resultado entre el grupo de padres y profesionales es prácticamente similar al obtenido entre profesionales y usuarios/as (grupo con el mayor grado de congruencia).

La baja congruencia general encontrada entre los tres grupos, puede deberse a que únicamente hay coincidencia por parte de todos en aquellos casos en los que el/la usuario/a muestre unas capacidades claras que permitan responder con claridad por parte de los tres agentes (personas con discapacidad con alta funcionalidad). Así mismo, la baja congruencia encontrada entre padres e hijos/as muestra que los padres conocen relativamente poco a sus hijos/as en cuanto a sexualidad se refiere, promovidas estas actitudes por mitos atribuidos a este colectivo, falsas creencias respecto a las necesidades sexuales de sus hijos/as, influencias culturales y religiosas, etc.

Finalmente, por lo que se refiere al grado de acuerdo en cuanto al beneficio de proporcionar/recibir educación afectivo-sexual, se mostró una alta congruencia entre los tres grupos que coinciden 
en considerar beneficiosa la formación en sexualidad de las personas con DFI. El beneficio para el/la propio/a usuario/a de esa educación efectivo-sexual ha sido demostrado por otros investigadores (Gil-Llario, Morell-Mengual, Ballester-Arnal, \& Díaz-Rodríguez, 2018; Rojas, Haya, \& Lázaro, 2015). De nuevo, los grupos que obtuvieron mayor acuerdo de opiniones fueron los/as usuarios/as con los profesionales, seguido por el par de padres-profesionales y casi en paralelo, el de usuarios-padres. Estas buenas actitudes por parte de los tres grupos y sobre todo, en la comparación entre usuarios y padres, puede deberse a una disminución progresiva de los prejuicios a saber del tema y a no limitar el conocimiento en sexualidad en beneficio de la prevención del abuso sexual tal como señalan -entre otros- Doughty \& Kane (2010). Además, la media de edad de nuestra muestra era relativamente joven lo que puede indicar que las actitudes de los padres también van adaptándose a las nuevas generaciones y en muchos casos, están influenciadas por las actitudes del resto de familiares de las personas con DFI (hermanos/as, cuñados/as, primos/as, etc.).

En resumen, los datos reflejan la necesidad de mejorar la comunicación en temas de sexualidad entre los tres agentes implicados en la educación de las personas con DFI (usuarios, padres y profesionales). Dada la buena predisposición por parte de todos los evaluados en este estudio para proporcionar/recibir educación afectivo-sexual, se abre un abanico de posibilidades para implementar talleres para padres, formación para profesionales, cursos para personas con DFI, etc. De este modo, se generará mayor confianza entre los tres agentes a la hora de hablar sobre sexualidad, se incrementarán los conocimientos sobre esta importante área del desarrollo y se facilitará la autoprotección de las personas con diversidad funcional intelectual de riesgos de abuso sexual.

\section{REFERENCIAS}

Aunos, M., \& Feldman, M. A. (2002). Attitudes towards sexuality, sterilization and parenting rights of persons with intellectual disabilities. Journal of Applied Research in Intellectual Disabilities, 15(4), 285-296. https://doi.org/10.1046/j.1468-3148.2002.00135.x

Azzopardi-Lane, C., \& Callus, A. M. (2015). Constructing sexual identities: People with intellectual disability talking about sexuality. British Journal of Learning Disabilities, 43(1), 32-37. https://doi.org/10.1111/bld.12083

Byrne, G. (2017). Prevalence and psychological sequelae of sexual abuse among individuals with an intellectual disability. Journal of Intellectual Disabilities, 1-17. https://doi.org/10.1177/1744629517698844

Cuskelly, M., \& Bryde, R. (2004). Attitudes towards the sexuality of adults with an intellectual disability: parents, support staff, and a community sample. Journal of Intellectual and Developmental Disability, 29(3), 255-264. https://doi.org/10.1080/13668250412331285136

Doughty, A. H., \& Kane, L. M. (2010). Teaching abuse-protection skills to people with intellectual, disabilities: A review of the literature. Research in Developmental Disabilities, 31(2), 331-337. https://doi.org/10.1016/j.ridd.2009.12.007

Evans, D. S., McGuire, B. E., Healy, E., \& Carley, S. N. (2009). Sexuality and personal relationships for people with an intellectual disability. Part II: Staff and family carer perspectives. Journal of Intellectual Disability Research, 53(11), 913-921. https://doi.org/10.1111/j.13652788.2009.01202.x

Frawley, P., \& Wilson, N. J. (2016). Young People with Intellectual Disability Talking About Sexuality Education and Information. Sexuality and Disability, 34(4), 469-484. https://doi.org/10.1007/s11195-016-9460-x

Gil-Llario, M.D., Ballester-Arnal, R. y Morell-Mengual, V. (2017). Evaluación de la sexualidad de personas con diversidad funcional en el marco de un programa de educación afectivo sexual, 3rd 
International Congress of Clinical and Health Psychology on Children and Adolescents, Sevilla, 18th November

Gil-Llario, M. D., Morell-Mengual, V., Ballester-Arnal, R., \& Díaz-Rodríguez, I. (2018). The experience of sexuality in adults with intellectual disability. Journal of Intellectual Disability Research, 62(1), 72-80. https://doi.org/10.1111/jir.12455

Isler, A., Beytut, D., Tas, F., \& Conk, Z. (2009). A study on sexuality with the parents of adolescents with intellectual disability. Sexuality and Disability, 27(4), 229-237. https://doi.org/10.1007/s11195-009-9130-3

Lafferty, A., McConkey, R., \& Simpson, A. (2012). Reducing the barriers to relationships and sexuality education for persons with intellectual disabilities. Journal of Intellectual Disabilities, 16(1), 29-43. https://doi.org/10.1177/1744629512438034

Löfgren-Mårtenson, L. (2004). "May I?" About Sexuality and Love in the New Generation with Intellectual Disabilities. Sexuality and Disability, 22(3), 197-207. https://doi.org/10.1023/B:SEDI.0000039062.73691.cb

Löfgren-Mårtenson, L. (2012). "I Want to Do it Right!" A Pilot Study of Swedish Sex Education and Young People with Intellectual Disabilities. Sexuality and Disability, 30(2), 209-225. https://doi.org/10.1007/s11195-011-9239-z

McCabe, M. P., \& Cummins, R. A. (1996). The Sexual Knowledge, Experience, Feelings and Needs of People with Mild Intellectual Disability. Education and Training in Mental Retardation and Developmental Disabilities, 31(1), 13-21. https://doi.org/10.2307/23879019

Povilaitiene, N., \& Radzeviciene, L. (2013). Parents' and teachers' attitude to family planning of adolescents with mild intellectual disabilities in the context of conceptions of gender and sexuality. Social Welfare: Interdisciplinary Approach, 2(3), 8-18.

Rohleder, P. (2010). Educators' ambivalence and managing anxiety in providing sex education for people with learning disabilities. Psychodynamic Practice, 16(2), 165-182. https://doi.org/10.1080/14753631003688100

Rojas, S., Haya, I., \& Lázaro, S. (2015). Necesidades afectivo-sexuales en personas con discapacidad intelectual. Claves para construir propuestas formativas desde la experiencia subjetiva. Revista Española de Discapacidad, 2(3), 41-54.

Schaafsma, D., Kok, G., Stoffelen, J. M. T., \& Curfs, L. M. G. (2014). Identifying Effective Methods for Teaching Sex Education to Individuals With Intellectual Disabilities: A Systematic Review. The Journal of Sex Research, 52(4), 412-432. https://doi.org/10.1080/00224499.2014.919373

Schwartz, R. J., \& Robertson, R. E. (2018). A Review of Research on Sexual Education for Adults With Intellectual Disabilities. Career Development and Transition for Exceptional Individuals, 1-10. https://doi.org/10.1177/2165143418756609

Thompson, V. R., Stancliffe, R. J., Broom, A., \& Wilson, N. J. (2014). Barriers to sexual health provision for people with intellectual disability: A disability service provider and clinician perspective. Journal of Intellectual and Developmental Disability, 39(2), 137-146. https://doi.org/10.3109/13668250.2014.898742

Thompson, V. R., Stancliffe, R. J., Broom, A., \& Wilson, N. J. (2016). Clinicians' use of sexual knowledge assessment tools for people with intellectual disability. Journal of Intellectual and Developmental Disability, 41(3), 243-254. https://doi.org/10.3109/13668250.2016.1164303 
